\title{
APLICACIÓN DE ESTUDIOS GEOQUÍMICOS SOBRE ARTEFACTOS DE OBSIDIANA PROCEDENTES DE CONTEXTOS ESTRATIGRÁFICOS Y SUPERFICIALES EN ALDEA BELEIRO, SUDOESTE DE CHUBUT (PATAGONIA, ARGENTINA)
}

\author{
ANALÍA CASTRO ESNAL ${ }^{\mathrm{a}}$, CHARLES STERN ${ }^{\mathrm{a}}$ CECILIA PÉREZ DE MICOU ${ }^{c}$
}

\begin{abstract}
RESUMEN
En este trabajo se presentan nuevos datos producto de la aplicación del análisis de ICP-MS en muestras de artefactos de obsidianas hallados en depósitos arqueológicos estratificados excavados en la cueva principal (CP1) del sitio Casa de Piedra, Estancia Roselló, en Aldea Beleiro (SO Chubut, Patagonia, Argentina) y en sitios de superficie del área de estudio. Entre los resultados se observa que en CP1 predomina la obsidiana de la fuente Pampa del Asador (PDA) (Santa Cruz, Argentina), aunque se destaca el hallazgo de un artefacto de obsidiana del valle del Río Cisnes (CIS) (Aisén, Chile) en un componente fechado en 7.700 años AP. Asimismo, se presenta el caso más austral, hasta el momento conocido, de obsidiana de Sacanana (S1), recuperada en Arenal Solsona, sitio de superficie cercano a CP1. En el trabajo se discute esta información integrándola con la conocida hasta el momento para la provincia de Chubut (Argentina) y Aisén (Chile). De esta manera, se realiza una síntesis y puesta al día del estado de la cuestión y se discuten las implicancias de los nuevos resultados para el conocimiento de las estrategias tecnológicas y circuitos de movilidad de los cazadores recolectores, tomando en cuenta un amplio marco cronológico que abarca desde el Holoceno temprano hasta los tiempos de contacto hispano-indígena.
\end{abstract}

PALABRAS CLAVE: circulación de obsidiana, análisis geoquímicos por ICP-MS, tendencias temporales, Chubut, Argentina.

GEOCHEMICAL STUDIES OF ARCHAEOLOGICAL OBSIDIAN ARTIFACTS FROM BOTH STRATIGRAPHIC AND SURFACE CONTEXTS IN ALDEA BELEIRO VILLAGE, SOUTHWESTERN CHUBUT (PATAGONIA, ARGENTINA)

\author{
ABSTRACT \\ In this work, new data are presented resulting from the application of ICP-MS analysis of \\ a Consejo Nacional de Investigaciones Científicas y Técnicas (CONICET). Instituto Nacional de Antropología y Pensamiento \\ Latinoamericano (INAPL). 3 de Febrero 1378, C1426BJN, Buenos Aires, Argentina. analiacastro@gmail.com \\ b University of Colorado. Department of Geological Sciences, University of Colorado, Boulder, Colorado, 80309-0399, USA. Charles. \\ Stern@Colorado.edu \\ c Consejo Nacional de Investigaciones Científicas y Técnicas (CONICET). Universidad de Buenos Aires. Instituto Nacional de Antropología \\ y Pensamiento Latinoamericano (INAPL). 3 de Febrero 1378, C1426BJN, Buenos Aires, Argentina. c.perezdemicou@yahoo.com.ar
}


samples of obsidian artifacts found in layered archaeological deposits excavated in the main cave (CP1) of Casa de Piedra, Estancia Roselló, Aldea Beleiro (SW Chubut, Patagonia, Argentina) and in surface sites nearby. The results show that the predominant obsidian in CP1 is from the Pampa del Asador source (PDA) (Santa Cruz, Argentina), although the finding of one obsidian artifact from Río Cisnes (CIS) (Aisén, Chile) in a component dated $\sim 7.700$ years BP stands out. Also, the southernmost case of obsidian from Sacanana (S1) has been recovered in Arenal Solsona, a surface site near CP1. These new results and the previous information from Chubut (Argentina) and Aisén (Chile) are discussed. Thus, a summary and update of the state of the information is presented and the implications of the new findings for understanding the technological strategies and mobility circuits of hunter-gatherers are discussed, taking into account a broad chronological framework ranging from the early Holocene until the times of contact.

KEY WORDS: obsidian circulation, geochemical analysis by ICP-MS, temporal trends, Chubut, Argentina.

\section{INTRODUCCIÓN}

La distribución de artefactos líticos de materias primas extra regionales ha sido utilizada como un indicador de movilidad e interacción entre grupos nómades de Patagonia central (Gómez \& Stern, 2005; Bellelli et al. 2006; Méndez et al. 2008/2009, 2012; Castro Esnal et al. 2011; Stern et al. 2013; entre otros). En el caso de la provincia de Chubut, la caracterización geoquímica de obsidianas ha señalado tres fuentes principales: 1) Sacanana (Cerro Guacho), con una amplia representación del tipo S1 en sitios del norte de la provincia (Stern et al. 2000; Bellelli \& Pereyra, 2002; Gómez \& Stern, 2005; Bellelli et al. 2006; Pérez \& Castro Esnal, 2007; Stern et al. 2007; Castro Esnal et al. 2011; Favier et al. 2009; entre otros); 2) Telsen (Sierra Negra), distribuida especialmente en el noreste de la provincia de Chubut (Stern et al. 2000; Gómez \& Stern, 2005; Favier et al. 2009); y 3) Pampa del Asador (provincia de Santa Cruz), hallada principalmente en sitios ubicados hacia el sur del paralelo 44 (Stern, 1999, 2004; Espinosa \& Goñi, 1999; Belardi et al. 2006; Castro Esnal et al. 2011; Stern et al. 2013; Reyes et al. 2015; entre otros).

Sin embargo, este esquema de distribución aparentemente relacionado con la cercanía relativa a cada fuente, se ve complejizado con el hallazgo de obsidianas en sitios muy alejados de sus respectivas fuentes, como es el caso del reconocimiento de obsidiana de Pampa del Asador en la costa norte de Chubut (Gómez \&
Stern, 2005), de obsidiana de Sacanana en el sur de esa provincia (Castro Esnal et al. 2011) y de Sacanana y Telsen en Alto Cisnes (Aisén, Chile) (Stern et al. 2013). Además, la presencia conjunta de distintos tipos de obsidiana en sitios arqueológicos relevados en zonas de caminos o rutas de movilidad conocidas por ser transitadas por los tehuelches de momentos de contacto, ha permitido plantear posibles corredores de circulación humana y transporte de artefactos a grandes distancias durante el Holoceno tardío (Castro Esnal et al. 2011; Stern et al. 2013; Castro Esnal, 2014).

En este trabajo se presentan nuevos resultados de los análisis geoquímicos de artefactos de obsidiana recuperados en estratigrafía en la cueva principal (CP1) del sitio Casa de Piedra de Roselló en Aldea Beleiro (SO de Chubut) y de otros sitios de superficie aledaños. La integridad estratigráfica de $\mathrm{CP} 1$ brinda la posibilidad de realizar análisis sobre obsidianas ubicadas en una secuencia continua de componentes que comienza en los inicios de ocupación del área (circa 7700 años AP) y culmina en los momentos históricos recientes. Cabe destacar la importancia de esta información dado que la existente hasta el momento para la provincia de Chubut se ha producido principalmente a partir de recolecciones superficiales sin información cronológica.

\section{PRESENTACIÓN DEL ÁREA DE ESTUDIO}

Los estudios arqueológicos en el área de 
Aldea Beleiro comenzaron en el año 2009. El sitio Casa de Piedra (CP) $\left(45,3^{\circ} \mathrm{S} ; 71,2^{\circ} \mathrm{O}\right.$ ) (Pérez et al. 2013) está ubicado en la Estancia Roselló, en Aldea Beleiro, sudoeste de la provincia de Chubut, en el límite entre la estepa -Distrito Occidental- y el bosque -Distrito Subandino(León et al. 1998; Soriano, 1956; Soriano et al. 1983), aproximadamente a $5 \mathrm{~km}$ del límite internacional con la República de Chile (Pérez et al. 2013) (Fig. 1). Se emplaza en un cañadón, atravesado por el arroyo Nirihuao, que comunica el Puesto Grande de la Estancia Mercedes Roselló con el comienzo de la Estancia El Ñire. Este lugar es conocido como El Portezuelo, ubicado en un macizo rocoso de ignimbrita correspondiente a la formación Carren Leufú (Dal Molin, 1998). Se trata de un sitio compuesto por tres cuevas ubicadas a unos $12 \mathrm{~m}$ de altura sobre el mallín por el que discurre el arroyo Nirihuao y a unos 100 $\mathrm{m}$ de distancia de éste. La cueva principal (CP1) presenta aproximadamente $9 \mathrm{~m}$ de profundidad y, como toda cueva o abrigo profundo, es una trampa sedimentaria con escasa conexión con el exterior cercano: no crecen en ella arbustos ni pastos, que sí están presentes en sus alrededores. La orientación de su boca la protege de los vientos predominantes del oeste; recibe luz natural, aunque su intensidad decrece hacia su interior. La protección de las precipitaciones se prolonga varios metros fuera de la boca (Pérez et al. 2013). Además de la cueva principal (CP1), el sitio presenta otras dos cuevas pequeñas con arte rupestre -CP2 y CP3-, localizadas en una cota superior (Fig. 2).

Hasta el momento se han excavado por décapage dos cuadrículas de 1 x $1 \mathrm{~m}$ en la cueva principal (CP1) alcanzando 1,20 m de profundidad. Se identificaron nueve niveles naturales continuos, arqueológicamente fértiles (material lítico y faunístico). El último nivel presenta escasos materiales antrópicos en su cumbre, y a medida que se profundiza se torna completamente estéril ${ }^{1}$. Entre los hallazgos recuperados, se destaca, por su frecuencia, el material lítico: 8.850 desechos de talla, 160 núcleos y 20 instrumentos (Pérez et al. 2014a; Castro Esnal et al. 2016). Los fechados realizados hasta el momento en CP1 indican una ocupación desde al menos $7719 \pm$ 51 años AP (Castro Esnal et al. 2016), siendo éste el fechado más temprano conocido para la zona. Las materias primas representadas en los conjuntos líticos de toda la columna estratigráfica muestran el uso predominante de una roca silícea local presente en la Formación Carren Leufú. No obstante, en todas las capas, se hallaron artefactos de obsidiana.

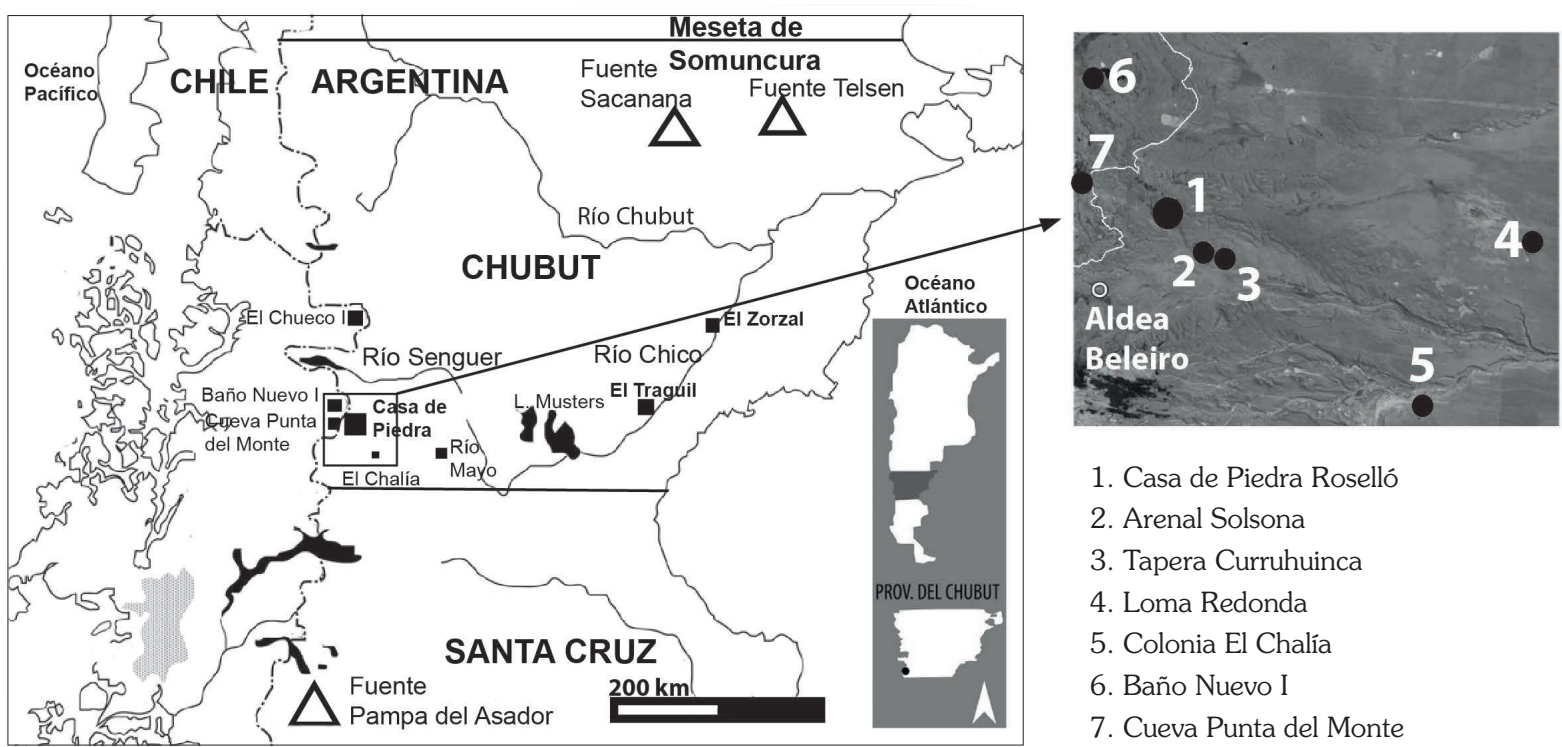

Fig. 1. Ubicación del área de estudio y de los sitios arqueológicos y fuentes de obsidiana mencionados en el texto.

1 Se estima llegar a la roca base en el próximo trabajo de campo 


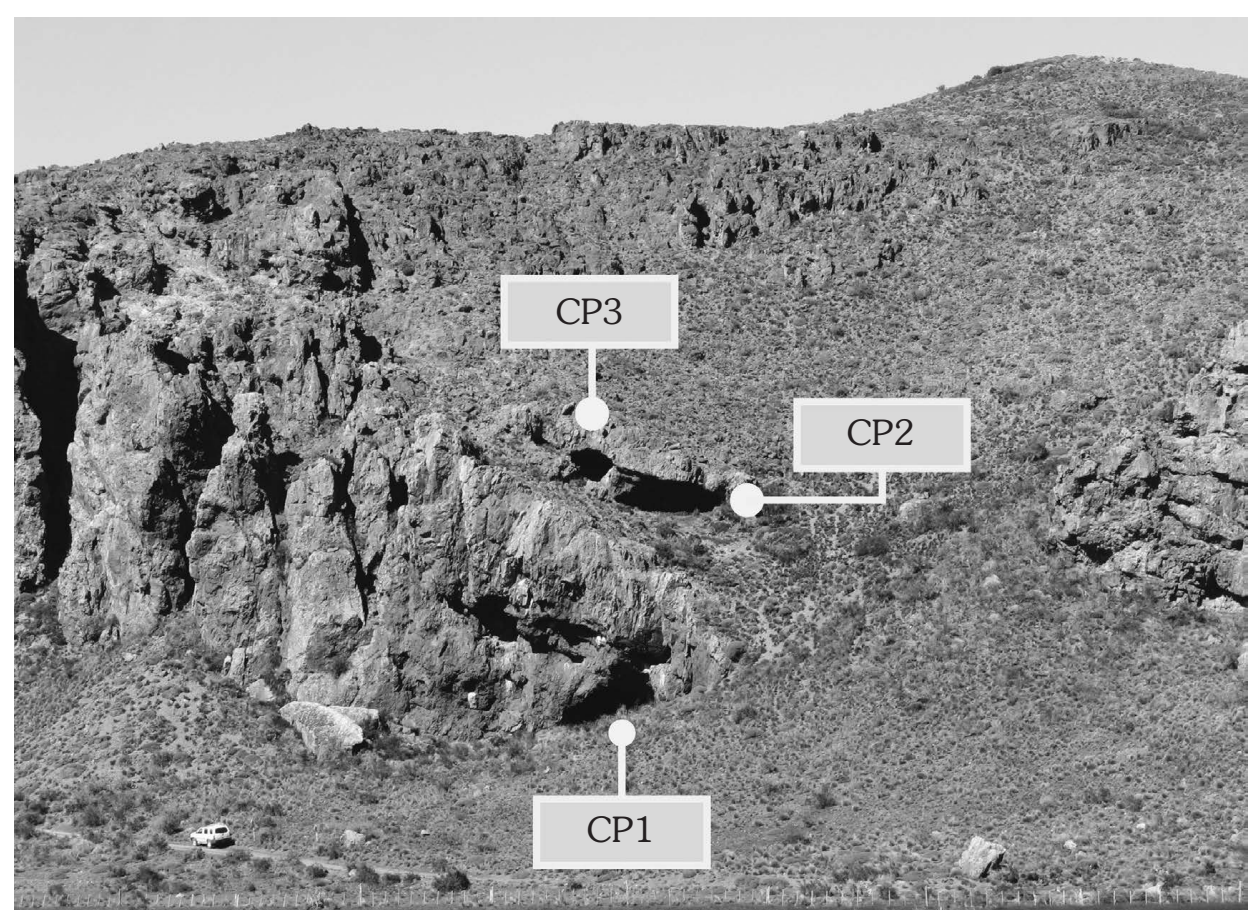

Fig. 2. Vista panorámica de Casa de Piedra de Roselló (Foto: M. L. Funes).

A una distancia aproximada de $8 \mathrm{~km}$ de $\mathrm{CP}$, también sobre el curso del arroyo Nirihuao, se ha relevado un sitio de superficie denominado

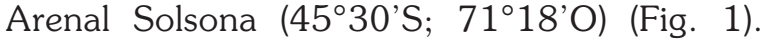
Se trata de una hoyada de deflación en donde el viento ha dejado al descubierto numerosos artefactos líticos $(n=354)$, en su mayoría desechos de talla, de una gran variedad de materias primas, incluyendo la obsidiana. En este sitio se realizó una recolección sistemática de la totalidad de los artefactos expuestos en superficie en un área de $\sim 2.800 \mathrm{~m}^{2}$ (Fig. 3).

Cerca de este sitio, aproximadamente a $2,5 \mathrm{~km}$, se han relevado los restos de un asentamiento de principios de siglo XX: Tapera Curruhuinca (Fig. 1). Este sitio presenta escasos materiales líticos en superficie $(n=5)$. Por último, más alejado de esta área, a $55 \mathrm{~km}$ aproximados de CP, en el sitio Loma Redonda (Fig. 1), se ha relevado también un sitio de momentos de contacto en el que se ha registrado la presencia de una gran estructura de piedra de forma circular y escaso material lítico $(n=16)$ en superficie (Pérez et al. 2014b). Estos últimos dos sitios aún no han sido trabajados sistemáticamente por lo que se presentan aquí sólo los datos de procedencia de las muestras de obsidiana halladas en superficie.

\section{LOS ARTEFACTOS DE OBSIDIANA DEL SUDOESTE DE CHUBUT Y ÁREAS VECINAS}

En el sudoeste de Chubut los datos sobre procedencia de obsidiana se circunscriben, hasta el momento, a lo conocido para los sitios de superficie estudiados en la Colonia El Chalía (4543'S; $70^{\circ} 55^{\prime}$ O) (Fig. 1). Estos resultados señalan a la obsidiana de Pampa del Asador como la única obsidiana que habría sido utilizada en el área, con excepción de dos casos de fuente desconocida (Castro Esnal et al. 2011). Recientemente, en latitudes aproximadamente similares hacia el este, se han analizado artefactos de obsidiana halladas en sitios de la cuenca del Lago Musters, que también provienen en su totalidad de la fuente de Pampa del Asador (Reyes et al. 2015).

Esto es coherente con los datos producidos por los trabajos arqueológicos realizados en latitudes similares en la región vecina de Aisén, Chile (Stern et al. 2013). Allí, el estudio de 
obsidianas en el sitio Baño Nuevo 1, cueva ubicada en las estepas abiertas del curso alto del río Nirehuao (Fig. 1), muestra el uso predominante de obsidiana de Pampa del Asador, con excepción de cuatro casos de fuente desconocida. Estos últimos cuatro casos fueron hallados en los componentes medio (8.770-5.660 años cal AP) y temprano (10.700-8.770 años cal. AP) del sitio (Méndez et al. 2008/2009; Méndez et al. 2012). Muy cerca de Baño Nuevo 1, en el sitio Cueva de Punta del Monte (Fig. 1), han sido analizadas muestras de obsidiana halladas en superficie que también fueron determinadas como procedentes de Pampa del Asador (Nuevo Delaunay et al. 2013).

Hacia el norte, en el curso superior del río Cisnes, el sitio El Chueco 1 evidencia la presencia de obsidiana de Pampa del Asador hacia los 10.180 años cal AP y entre los 5.520 y 5.400 años cal AP, pero además allí se ha registrado una muestra de obsidiana de Telsen hacia los 10.180 años cal AP (Reyes et al. 2007; Stern et al. 2013). En esta misma zona han sido halladas obsidianas en superficie procedentes de Sacanana y Telsen. Por último, también se observa tanto en superficie como en estratigrafía la presencia de escasos artefactos de una obsidiana local de menor calidad (denominada CIS) (Méndez et al. 2008/9; Méndez et al. 2012).

La ocurrencia conjunta de obsidianas provenientes de distintas fuentes también ha sido registrada en los sitios de superficie $\mathrm{El}$ Tragüil (45.1 $\left.{ }^{\circ} \mathrm{S} ; 67.4^{\circ} \mathrm{O}\right)$ y Puesto El Zorzal (44.3 $\left.{ }^{\circ} \mathrm{S} ; 66.5^{\circ} \mathrm{O}\right)$ ubicados en el Valle del Río Chico, sudeste de la provincia de Chubut (Fig. 1). En estos sitios los artefactos de obsidiana se presentan en bajísima frecuencia y provienen tanto de Pampa del Asador como de Sacanana (Stern et al. 2013). La obsidiana del tipo S1 del sitio El Tragüil constituía hasta ahora la evidencia más austral de esta variedad. Más hacia el sur, en el noroeste de la provincia de Santa Cruz, recientemente se han realizado estudios geoquímicos de muestras de obsidiana provenientes de numerosos sitios arqueológicos y sólo se ha hallado obsidiana de Pampa del Asador, con excepción de dos muestras de obsidiana gris de fuente desconocida (Stern, 1999; Fernández et al. 2015).

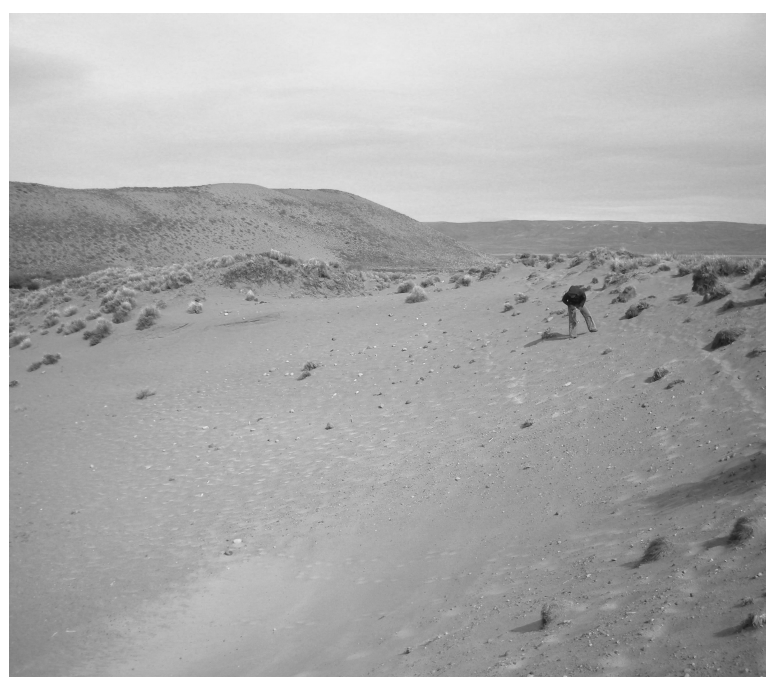

Fig. 3. Sitio Arenal Solsona. Foto: A. Castro.

\section{METODOLOGÍA}

Se seleccionaron muestras representativas de las distintas capas de CP1 y de los sitios de superficie cercanos (Arenal Solsona, Tapera Curruhuinca y Loma Redonda). Las muestras fueron elegidas según su peso $(>1 \mathrm{~g})$ y luego se procuró que estuvieran representadas todas las variedades macroscópicamente disímiles (en cuanto a su color, brillo, corteza, translucidez, etc.).

En total se pudieron analizar 16 muestras (de un total de 18 enviadas): 11 artefactos de obsidiana hallados en distintas capas de CP1; 3 de Arenal Solsona; 1 de Tapera Curruhuinca y 1 de Loma Redonda.

El análisis se llevó a cabo en la Universidad de Colorado (Boulder, EE.UU), donde las muestras fueron molidas a un polvo muy fino el cual fue disuelto en ácido fluorhídrico. Posteriormente, fueron analizadas por medio de ICP-MS (espectrometría de masa por plasma iónicamente acoplado, usando un instrumento tipo ELAN DCR-E) para obtener la composición de elementos traza en partes por millón ( $\mathrm{ppm}$ ). Los análisis de los estándares internos indican que la precisión para todos los elementos es mayor al $\pm 10 \%$. Los resultados obtenidos fueron comparados gráficamente con aquellos que se disponen de muestras geológicas de las fuentes de Patagonia central (Fig. 4). 


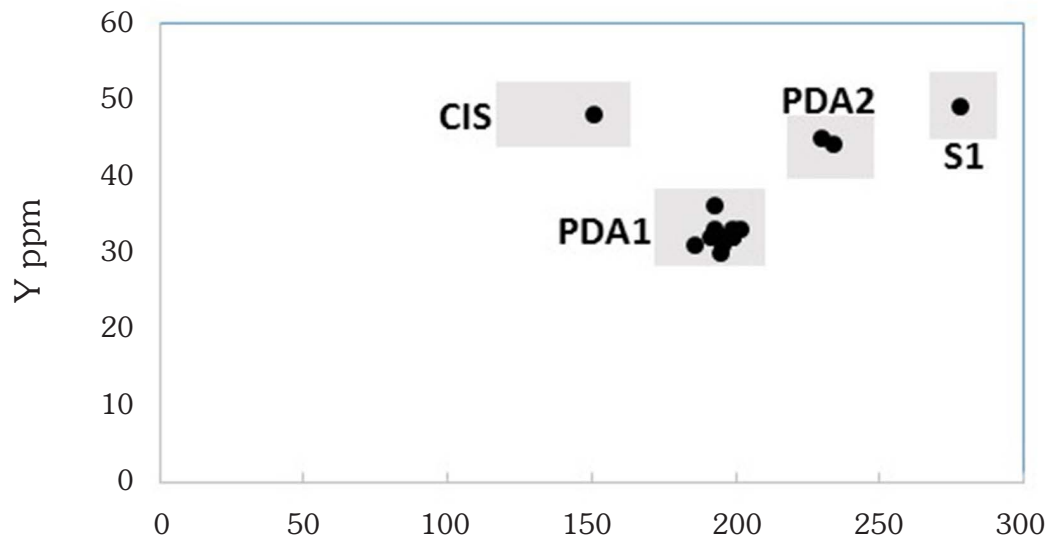

$\mathrm{Rb}$ ppm
Fig. 4. Rb versus $\mathrm{Y}$ (en ppm) para las muestras de CP1 (círculos; ver Tabla 2). Los recuadros grises indican el rango de composiciones para las muestras geológicas de los distintos tipos de obsidiana del sur de la Patagonia (Stern, 2004; Méndez et al. 2012; Fernández et al. 2015).

\section{RESULTADOS}

En la Tabla 1 se presentan los tipos de obsidiana identificados en Casa de Piedra 1 (CP1), Arenal Solsona, Tapera Curruhuinca y Loma Redonda. En el caso de CP1, se indican también los fechados radiocarbónicos obtenidos hasta el momento en las capas de donde proviene cada muestra. En la Tabla 2 se detallan los elementos químicos determinados para cada caso. Se observa una clara predominancia del tipo PDA 1 de Pampa del Asador. Sin embargo, en la Capa 6 de CP1 fechada en 7719 \pm 51 años AP (Castro Esnal et al. 2016), llama la atención la presencia de una obsidiana del tipo CIS (Río Cisnes). Del mismo modo, se destaca la presencia de obsidiana del tipo S1 en el sitio de superficie Arenal Solsona, ya que representa el hallazgo más austral de obsidiana de este tipo.

Cabe destacar que la obsidiana en CP1 se presenta siempre en bajas proporciones con respecto a las materias primas locales. Las mayores proporciones relativas de obsidiana se registran en las capas 5, 6, 7 y 8 (Tabla 3). En todos los casos la categoría que predomina ampliamente en los conjuntos de obsidiana es la de desechos de talla (Tabla 4). Son mayoritariamente desechos de tamaño pequeño o mediano/pequeño (sensu Bagolini modificado en Aschero, 1975, 1983) que representan las últimas etapas del proceso de talla. Con respecto a la presencia de núcleos, sólo se recuperó un pequeño núcleo bipolar, lo que daría cuenta de un uso intensivo de esta materia prima. En cuanto a los instrumentos formatizados (13 en total), es importante la representación de artefactos con talla bifacial extendida (8 en total). En cuatro casos se trata de puntas de proyectil (una de la Capa 4 y tres de la Capa 6), sin embargo sólo dos de ellas (recuperadas en la Capa 6) se presentan enteras (Fig. 5), el resto de los artefactos bifaciales no puede ser asignado a ningún grupo tipológico por su estado de fragmentación. El conjunto de instrumentos está integrado además por dos raspadores, una raedera y una muesca (Tabla 5).

Del mismo modo, las obsidianas halladas en superficie en el sitio Arenal Solsona son mayoritariamente desechos de talla. El total del conjunto de obsidianas recuperado en este sitio está compuesto por 56 desechos, un filo natural con rastros complementarios y un fragmento de filo. Por último, para el caso del sitio Tapera Curruhuinca se trata de un único hallazgo aislado (un desecho de talla) y para el de Loma Redonda de un raspador y de una lasca que presenta un filo natural con rastros complementarios.

\section{DISCUSIÓN}

Tendencias temporales en el uso de obsidiana en el sudoeste de Chubut

Los análisis de procedencia realizados sobre artefactos de obsidiana ubicados en contextos estratigráficos permiten discutir y comparar 
Tabla 1. Tipos de obsidiana identificados en el área de estudio.

\begin{tabular}{|c|c|c|c|c|}
\hline Sitio & Muestra \# & Procedencia & Fuente & $\begin{array}{l}\text { Fechados }{ }^{14} \mathrm{C} \text { AMS } \\
\text { (Pérez et al. 2013; } \\
\text { Castro et al. 2016) }\end{array}$ \\
\hline Casa de Piedra 1 & 1 & superficie & PDA2 & \\
\hline Casa de Piedra 1 & 2 & Capa 1 & PDA1 & $493 \pm 34$ años AP \\
\hline Casa de Piedra 1 & 3 & Capa 3 & PDA1 & \\
\hline Casa de Piedra 1 & 4 & Capa 4 & PDA2 & $5682 \pm 46$ años AP \\
\hline Casa de Piedra 1 & 7 & Capa 4 & PDA1 & $5682 \pm 46$ años AP \\
\hline Casa de Piedra 1 & 8 & Capa 4 & PDA1 & $5682 \pm 46$ años AP \\
\hline Casa de Piedra 1 & 9 & Capa 5 & PDA1 & \\
\hline Casa de Piedra 1 & 10 & Capa 6 & CIS & $7719 \pm 51$ años $A P$ \\
\hline Casa de Piedra 1 & 11 & Capa 6 & PDA1 & $7719 \pm 51$ años AP \\
\hline Casa de Piedra 1 & 12 & Capa 6 & PDA1 & $7719 \pm 51$ años $A P$ \\
\hline Casa de Piedra 1 & 13 & Capa 6 & PDA1 & $7719 \pm 51$ años AP \\
\hline Arenal Solsona & 14 & superficie & $\mathrm{S} 1$ & \\
\hline Arenal Solsona & 15 & superficie & PDA1 & \\
\hline Arenal Solsona & 16 & superficie & PDA1 & \\
\hline Tapera Curruhuinca & 17 & superficie & PDA1 & \\
\hline Loma Redonda & 18 & superficie & PDA1 & \\
\hline
\end{tabular}

Tabla 2. Resultados de los análisis geoquímicos aplicados en cada muestra.

\begin{tabular}{cccccccccccccccc}
\hline Lab \# & $\begin{array}{c}\text { Muestra } \\
\#\end{array}$ & Tipo & Ti* & Mn & Rb & Sr & $\mathrm{Y}$ & $\mathrm{Zr}$ & $\mathrm{Nb}$ & $\mathrm{Cs}$ & $\mathrm{Ba}$ & $\mathrm{Hf}$ & $\mathrm{Pb}$ & $\mathrm{Th}$ & $\mathrm{U}$ \\
\hline 9001 & 1 & PDA2 & 748 & 199 & 234 & 2 & 44 & 141 & 38 & 11,9 & 10 & 9,3 & 23,5 & 21,2 & 6,3 \\
9002 & 2 & PDA1 & 784 & 256 & 196 & 32 & 31 & 129 & 28 & 9,9 & 228 & 6,2 & 21,2 & 19,9 & 5,0 \\
9003 & 3 & PDA1 & 800 & 256 & 199 & 32 & 32 & 129 & 26 & 9,7 & 231 & 6,0 & 20,8 & 19,4 & 5,0 \\
9004 & 4 & PDA2 & 751 & 198 & 230 & 2 & 45 & 140 & 34 & 12,2 & 10 & 6,7 & 22,9 & 19,7 & 6,0 \\
9007 & 7 & PDA1 & 800 & 257 & 193 & 33 & 33 & 130 & 26 & 9,5 & 236 & 5,2 & 20,0 & 18,1 & 4,9 \\
9008 & 8 & PDA1 & 770 & 249 & 186 & 32 & 31 & 124 & 25 & 9,5 & 226 & 5,0 & 19,8 & 17,1 & 4,8 \\
9009 & 9 & PDA1 & 802 & 261 & 195 & 33 & 32 & 131 & 26 & 9,6 & 235 & 5,3 & 19,6 & 18,0 & 5,0 \\
9010 & 10 & Cisnes local & 983 & 497 & 151 & 8 & 48 & 278 & 26 & 5,5 & 488 & 8,7 & 16,6 & 17,9 & 4,4 \\
9011 & 11 & PDA1 & 780 & 256 & 193 & 33 & 36 & 131 & 25 & 9,6 & 233 & 5,2 & 19,6 & 17,4 & 4,8 \\
9012 & 12 & PDA1 & 789 & 252 & 192 & 32 & 32 & 129 & 33 & 9,4 & 227 & 7,1 & 19,2 & 19,6 & 5,0 \\
9013 & 13 & PDA1 & 748 & 250 & 195 & 31 & 30 & 126 & 25 & 9,5 & 222 & 5,1 & 18,9 & 17,0 & 4,9 \\
9014 & 14 & S1 Sacanana & 924 & 319 & 278 & 4 & 49 & 317 & 130 & 3,9 & 11 & 12,3 & 20,6 & 22,1 & 6,1 \\
9015 & 15 & PDA1 & 826 & 258 & 199 & 32 & 32 & 131 & 25 & 9,6 & 230 & 4,9 & 19,7 & 17,3 & 5,1 \\
9016 & 16 & PDA1 & 838 & 262 & 202 & 33 & 33 & 136 & 26 & 9,7 & 233 & 5,1 & 19,9 & 17,6 & 5,2 \\
9017 & 17 & PDA1 & 810 & 253 & 199 & 33 & 33 & 133 & 25 & 9,6 & 229 & 5,0 & 19,8 & 17,3 & 5,1 \\
9018 & 18 & PDA1 & 843 & 255 & 199 & 32 & 32 & 129 & 25 & 9,1 & 226 & 4,9 & 19,3 & 17,3 & 5,0 \\
\hline
\end{tabular}


Tabla 3. Cantidades relativas de obsidiana en CP1 (en estratigrafía) y en Arenal Solsona (en superficie).

\begin{tabular}{cccccc}
\hline Sitio / Procedencia & $\begin{array}{c}\text { Total } \\
\text { Obsidianas }\end{array}$ & $\begin{array}{c}\text { Total } \\
\text { Lítico }\end{array}$ & $\%$ & $\begin{array}{c}\text { Muestras } \\
\text { Analizadas }\end{array}$ & Fuentes \\
\hline Casa de Piedra 1 / Capa 0 & 1 & 38 & 2.63 & 0 & - \\
Casa de Piedra 1 / Capa 1 & 24 & 646 & 3.71 & 1 & PDA1 \\
Casa de Piedra 1 / Capa 2 & 25 & 951 & 2.62 & 0 & - \\
Casa de Piedra 1 / Capa 3 & 10 & 477 & 2.09 & 1 & PDA1 \\
Casa de Piedra 1 / Capa 4 & 165 & 4537 & 3.63 & 3 & 2 PDA1 \\
Casa de Piedra 1 / Capa 5 & 64 & 448 & 14.28 & 1 & PDA1 \\
Casa de Piedra 1 / Capa 6 & 235 & 1715 & 13.70 & 4 & 3 PDA1 \\
Casa de Piedra 1 / Capa 7 & 13 & 162 & 8.02 & 0 & CIS \\
Casa de Piedra 1 / Capa 8 & 5 & 21 & 23.80 & 0 & - \\
Arenal Solsona / Superficie & 58 & 354 & 16.38 & 3 & 2 PDA1 \\
\hline Total & 500 & 9349 & 6.42 & $\begin{array}{c}\text { del total de } \\
\text { obsidianas }\end{array}$ & \\
\hline
\end{tabular}

Tabla 4. Características de la composición artefactual de las obsidianas de CP1 por capa.

\begin{tabular}{lccccccccccc}
\hline Capa & Superf. & 0 & 1 & 2 & 3 & 4 & 5 & 6 & 7 & 8 & Total \\
\hline Instrumentos & 1 & 1 & 1 & 0 & 0 & 4 & 0 & 6 & 0 & 0 & 13 \\
Desechos & 1 & 0 & 22 & 25 & 10 & 161 & 64 & 229 & 13 & 5 & 530 \\
Núcleos & 0 & 0 & 1 & 0 & 0 & 0 & 0 & 0 & 0 & 0 & 1 \\
\hline Total & 2 & 1 & 24 & 25 & 10 & 162 & 64 & 235 & 13 & 5 & 544 \\
\hline
\end{tabular}

su uso en distintos momentos. Para el caso de $\mathrm{CP} 1$, se observa que la obsidiana de la fuente Pampa del Asador (ubicada a una distancia lineal aproximada de $270 \mathrm{~km}$ hacia el sur) está presente desde los momentos tempranos de ocupación del sitio y además es el tipo que predomina en toda la secuencia estratigráfica. Esta misma situación se presenta en el sitio próximo Baño Nuevo 1, en la región colindante de Aisén, Chile. No obstante, los resultados presentados en este trabajo señalan una diferencia en cuanto a la frecuencia de utilización de obsidiana a lo largo de toda la secuencia de ocupación de CP1: los componentes más antiguos muestran una mayor frecuencia relativa de esta materia prima con respecto a otras rocas (Tabla 3 ).

Además, en CP1 se observan diferencias temporales en cuanto a las fuentes de obsidiana

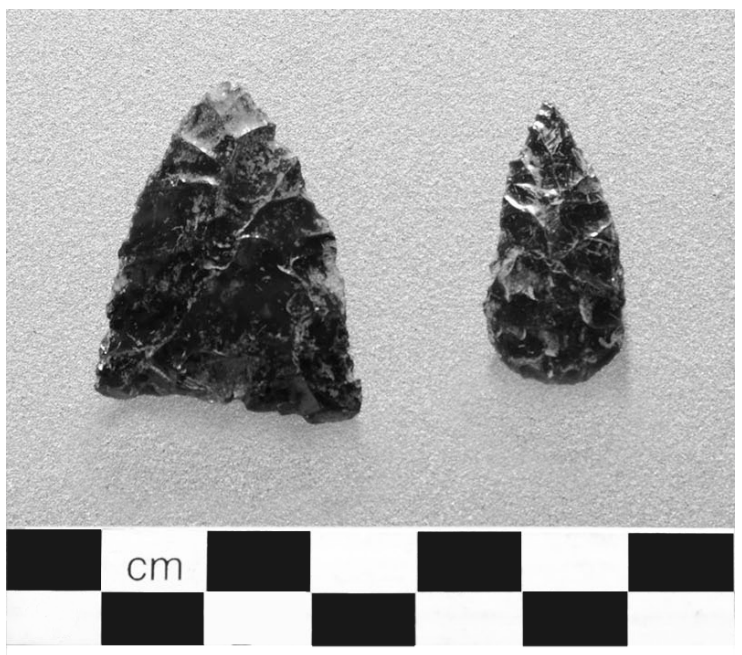

Fig. 5. Puntas de proyectil de obsidiana enteras de la Capa 6 de CP1. 
Tabla 5. Grupos Tipológicos de los instrumentos de obsidiana de CP1 por capa.

\begin{tabular}{lccccccccccc}
\hline & Superf. & 0 & 1 & 2 & 3 & 4 & 5 & 6 & 7 & 8 & Total \\
\hline Raspador & 1 & 1 & - & - & - & - & - & - & - & - & 2 \\
Punta de proyectil & - & - & - & - & - & 1 & - & 3 & - & - & 4 \\
Fragmento de filo & - & - & - & - & - & 1 & - & - & - & - & 1 \\
$\begin{array}{l}\text { Fragmento de artefacto } \\
\text { bifacial }\end{array}$ & - & - & - & - & - & 1 & - & 3 & - & - & 4 \\
Muesca & - & - & 1 & - & - & - & - & - & - & - & 1 \\
Raedera & - & - & - & - & - & 1 & - & - & - & - & 1 \\
\hline Total & 1 & 1 & 1 & - & - & 4 & - & 6 & - & - & 13 \\
\hline
\end{tabular}

representadas. En este sentido, se destaca la presencia de obsidiana del tipo CIS en el estrato fechado en $7719 \pm 51$ años AP. La ocurrencia de obsidianas de distintas fuentes también se presenta en Baño Nuevo 1, en donde Méndez y otros (2012) observan cuatro casos de obsidiana de fuente desconocida en los componentes más antiguos del sitio. Estos autores plantean la posibilidad de que tempranamente fueran utilizadas fuentes alternativas de obsidiana de diversa calidad $y$ que posteriormente ya no habrían sido seleccionadas debido a que presentaban una calidad para la talla menor en comparación con la de Pampa del Asador. El hecho de que en el componente tardío de Baño Nuevo 1 sólo se haya hallado obsidiana de PDA refuerza esta hipótesis (Méndez et al. 2012). Además de presentar ventajas en cuanto a su calidad, esta fuente se presenta disponible en una amplia área debido a que sus nódulos fueron transportados por procesos fluviales hasta algunos lugares relativamente alejados de la fuente, lo que incrementa su accesibilidad (Belardi et al. 2006).

Por otra parte, la presencia en el sudoeste de Chubut de obsidiana procedente del río Cisnes es un indicador de la existencia de algún tipo de vínculo, constatado al menos para los momentos en los que ambos tipos de obsidiana se observan de manera conjunta, entre estas dos zonas del oeste patagónico alejadas por aproximadamente $100 \mathrm{~km}$ de distancia. El hallazgo de obsidianas del tipo S1 (Sacanana) y T/SC (Telsen) en sitios de Alto Río Cisnes, tanto en contextos superficiales como estratigráficos (Stern et al. 2013), señalan también conexiones de esta zona de Chile con el noreste de la provincia de Chubut.

Siguiendo el modelo de poblamiento propuesto por Borrero (1994/1995) y las expectativas señaladas por Borrero y Franco (1997), Méndez y otros (2012) plantearon que la presencia de obsidiana de Pampa del Asador en los componentes tempranos de Baño Nuevo 1 (10.700 años cal AP) está señalando que ya para ese momento los grupos cazadores recolectores de la región transportaban rocas desde fuentes alejadas y por lo tanto tenían un conocimiento amplio del territorio, que no se condice con una etapa "exploratoria" sino con una de "colonización". Esta misma situación es factible de ser postulada para el caso de CP1, y además debe tomarse en cuenta también la posibilidad de que esta obsidiana fuera obtenida a través de contactos con otros grupos y no sólo por aprovisionamiento directo.

\section{Circuitos de movilidad de grupos} humanos para momentos tardíos

Los valles de los ríos habrían funcionado como corredores de circulación naturales, al ser caminos de menor costo, asociados con la disponibilidad de recursos básicos que facilitarían el desplazamiento (Borrero et al. 2008). El río Senguer nace en los lagos La Plata y Fontana, recibe los aportes de distintos arroyos de la zona y del río Mayo que surca de oeste a este todo el sudoeste de Chubut y que a su vez recibe también el aporte del arroyo Chalía (González, 1978). Luego, desemboca en los lagos Musters y Colhué Huapi y desde allí nace el río Chico (antiguamente también llamado Senguer), que 
actualmente culmina en el dique Ameghino (valle inferior del río Chubut) (Pérez de Micou et al. 2009a; Castro Esnal, 2014).

La cuenca del Senguer es mencionada en las fuentes históricas como vía de circulación de grupos humanos que conectaba oeste y este de la actual provincia de Chubut (Castro Esnal et al. 2007; Castro Esnal, 2014). A partir de la integración de la información etnohistórica con evidencias arqueológicas, el área del valle del río Chico ha sido interpretada como ruta o corredor por el que habrian circulado grupos de cazadores recolectores que se desplazaban a grandes distancias durante el Holoceno tardío (Castro Esnal, 2014).

El arroyo Nirihuao, forma parte de esta red hidrográfica ya que desemboca en el río Mayo, tributario del río Senguer. Tanto CP1 como el sitio de superficie Arenal Solsona se ubican en las márgenes de este arroyo, por lo que esta área arqueológica constituye la parte occidental de la cuenca del Senguer y su información se suma a la de las otras áreas arqueológicas conocidas sobre esta misma cuenca: El Chalía (Pérez et al. 2009b; Castro Esnal et al. 2011; Castro Esnal, 2014), Río Mayo (Pérez et al. 2009c; Aguerre et al. 2011), Lagos Musters y Colhue Huapi (Castro Esnal et al. 2007; Moreno et al. 2007; Moreno \& Pérez, 2010; Reyes et al. 2013; Reyes \& Peralta, 2014; Reyes et al. 2015) y Valle del Río Chico (Pérez et al. 2009; Castro Esnal, 2014).

La presencia de artefactos de obsidiana a grandes distancias con respecto a su fuente de procedencia ha sido constatada en sitios de superficie en el sur de Chubut que, a pesar de no presentar datos cronológicos absolutos, han sido asumidos como de momentos tardíos. En este trabajo se presentó el sitio Arenal Solsona, a $8 \mathrm{~km}$ de CP1, que presenta obsidiana de la fuente Sacanana (S1) (ubicada aproximadamente a $400 \mathrm{~km}$ lineales de distancia hacia el noreste). Este es, hasta el momento, el límite más austral de dispersión de esta fuente. Como se mencionó anteriormente hay además otros datos tardíos de ocurrencia conjunta de obsidiana de Pampa del Asador y Sacanana en sitios ubicados en el área del valle de Río Chico (Castro Esnal et al. 2011; Stern et al. 2013; Castro Esnal, 2014). Estos datos apoyan la hipótesis de una conexión cultural entre el sudoeste y el noreste de la provincia de Chubut, a través del valle del río Chico, al menos para los momentos tardíos.

\section{CONCLUSIÓN}

Los datos presentados en este trabajo aportan evidencia con respecto a la distribución de artefactos de obsidiana en la Patagonia central en un marco cronológico. A partir de ello, se discuten tendencias temporales con respecto al uso de esta materia prima, alóctona para el sudoeste de Chubut, que al integrarse con los datos conocidos en las áreas cercanas señalan: a) un uso más diversificado de fuentes de obsidiana para los primeros momentos de ocupación del área; b) para el caso de CP1 una variación en la frecuencia de uso de obsidiana para distintos momentos temporales, siendo mayor en los momentos previos a los $~ 5.600$ años AP (con predominancia de la fuente Pampa del Asador); y c) la incorporación de obsidianas de fuentes ubicadas a grandes distancias para los momentos tardíos representados por los sitios de superficie conocidos (Arenal Solsona, El Zorzal y El Tragüil). Esto último permite afinar las hipótesis sobre uso del espacio planteadas en trabajos anteriores y sustenta la idea de que la cuenca del Senguer ha sido un eje estructurador y facilitador del movimiento de grupos en el espacio patagónico.

\section{AGRADECIMIENTOS}

Las investigaciones en Aldea Beleiro han sido financiadas a través de subsidios otorgados por la UBA y el CONICET (UBACYT 868 y PIP084). Agradecemos especialmente la colaboración y el apoyo logístico brindado por la Comuna de Aldea Beleiro, especialmente a las familias Roselló, Solsona y Pérez del Barrio. A los evaluadores por sus aportes y sugerencias que contribuyeron a mejorar el manuscrito original. Todas las observaciones y conclusiones presentadas en este trabajo son responsabilidad de los autores.

\section{BIBLIOGRAFÍA}

Aguerre, A. M., Arrigoni, G. I., Iantanos, N., \& Andrieu, M. 
(2011). Hacia una comprensión de la antropodinamia de los cazadores-recolectores de los río Mayo y Guenguel, sur oeste de Chubut, Argentina. Trabajo presentado en las VIII Jornadas de Arqueología de la Patagonia, Malargüe.

Aschero, C. A. (1975). Ensayo para una clasificación morfológica de artefactos líticos. Informe. CONICET.

Aschero, C. A. (1983). Ensayo para una clasificación morfológica de artefactos líticos. Apéndices A y B. Buenos Aires: MS.

Belardi, J., Tiberi, P., Stern, C., \& Súnico, A. (2006). Al Este del Cerro Pampa: ampliación del área de disponibilidad de obsidiana de la Pampa del Asador (Provincia de Santa Cruz). Intersecciones en Antropología, 7, 27-36.

Bellelli, C., \& Pereyra, F. (2002). Análisis geoquímicos de obsidiana. Distribuciones de fuentes y artefactos arqueológicos en el Noroeste del Chubut (Patagonia Argentina). Revista Werken, 3, 99-118.

Bellelli, C., Pereyra, F., \& Carballido, M. (2006). Obsidian localization and circulation in northwestern Patagonia (Argentina): sources and archaeological record. En M. Maggetti \& B. Messiga (Eds.), Geomaterials in Cultural Heritage (pp. 421- 255). London: Geological Society of London, Special Publications 257.

Borrero, L. A. (1994/5). Arqueología de la Patagonia. Palimpsesto. Revista de Arqueología, 4, 9-69.

Borrero, L. A., \& Franco, N. (1997). Early Patagonian huntergatherers: Subsistence and technology. Journal of Anthropological Research, 53, 219-239.

Borrero, L. A., Charlin, J., Barberena, R., Martin F., Borrazzo, K., \& L'Heureux, L. (2008). Circulación humana y modos de interacción al sur del río Santa Cruz. En L. A. Borrero \& N. V. Franco (Comps.), Arqueología del extremo sur del continente americano. Resultados de nuevos proyectos (pp. 155-174). Buenos Aires: CONICET-IMHICIHU.

Castro Esnal, A., Pérez de Micou, C., Burry, L. S., \& Trivi de Mandri, M. (2007). Paleoambiente y etnohistoria en el lago Colhué Huapi. En Tras las huellas de la materialidad. XVI Congreso Nacional de Arqueología (Tomo III, pp. 333-337). San Salvador de Jujuy: Universidad Nacional de Jujuy.

Castro Esnal, A., Pérez de Micou, C., \& Stern, C. (2011). Circulación de obsidiana en Chubut, Patagonia Central, Argentina: uso de las materias primas extra-regionales como indicadores de movilidad e interacción entre grupos cazadores recolectores. Revista do Museo de Arqueología y Etnología, 21, 93-102.

Castro Esnal, A. (2014). Camino y Piedra. Rutas indígenas y arqueología en la provincia de Chubut. Buenos Aires: Fundación de Historia Natural Félix de Azara.

Castro Esnal, A., Casanueva M. L., Sacchi M., \& C. Pérez de Micou, C. (2016). Estudios arqueológicos en Aldea Beleiro, SO del Chubut, Argentina. Desde el primer poblamiento hasta el siglo XX. Revista del Museo de Antropología, 9(1), 7-12.

Dal Molin, C. (1998). Hoja Geológica 4572-IV ALTO RÍO SENGUER. Programa Nacional de Cartas Geológicas de la República Argentina. Boletín n²55. Buenos Aires: SEGEMAR.

Espinosa S. L., \& Goñi R. A. (1999). ¡Viven!: una fuente de obsidiana en la provincia de Santa Cruz. En Soplando en el viento... Actas de las Terceras Jornadas de Arqueología de la Patagonia (pp.177-188). Neuquén: Instituto Nacional de Antropología y Pensamiento Latinoamericano y Universidad Nacional del Comahue.

Favier Dubois, C., Stern, C., \& Cardillo, M. (2009). Caracterización de la distribución y tipos de obsidiana presentes en la costa rionegrina, Argentina. En M. Salemme, F. Santiago, M. Álvarez, E. Piana, M. Vásquez, E. Mansur (Eds.) Arqueología de la Patagonia. Una mirada desde el último confín, (pp. 349-359). Ushuaia: Editorial Utopías.

Fernandez, V., Stern, C. R., \& Leal, P. (2015). Obsidian from northwestern Santa Cruz province, Argentina. Quaternary International, 375, 44-54.

Gómez Otero, J., \& Stern, C. R. (2005). Circulación, intercambio y uso de obsidianas en la costa de la provincia del Chubut (Patagonia, Argentina) durante el holoceno tardío. Intersecciones en Antropología, 6, 93-108.

González, R. L. (1978). Descripción Geológica de las Hojas 49a, Lago Blanco y 49b, Paso Río Mayo, Provincia de Chubut. Boletín $N^{\circ}$ 154-55. Buenos Aires: Servicio Geológico Nacional.

León, R. J. C., Bran, D., Collantes, M., Paruelo, J. M., \& Soriano, A. (1998). Grandes Unidades de Vegetación de la Patagonia Extra Andina. Ecología Austral, 8: 125-144.

Méndez Melgar, C., Stern, C. R., \&. Reyes Báez, O. (2008/2009). Transporte de obsidianas a lo largo de los andes de Patagonia central (Aisén, Chile). CazadoresRecolectores del Cono Sur, 3, 51-68.

Méndez Melgar, C., Stern, C. R., Reyes, O., \& Mena, F. (2012). Early Holocene long-distance obsidian transport in central-south Patagonia. Chungara, Revista de Antropología Chilena, 44,(3), 363-375.

Moreno, E., Videla, B., Pérez Ruiz, H., Asencio, L. S., \&. 
Leonfort, V. (2007). Búsqueda de indicadores de diversificación económica prehistórica en la cuenca del lago Musters (Chubut, Argentina), primeros resultados. En F. Morello, M. Martnic, A. Prieto \& G. Bahamonde (Eds.), Arqueología de Fuego-Patagonia. Levantando piedras, desenterrando huesos...y develando arcanos (pp. 23-32). Punta Arenas: Ediciones CEQUA.

Moreno, E., \& Pérez Ruiz, H. (2010). Evidencias de utilización prehispánica de recursos fluviales en la cuenca del lago Musters (Chubut, Argentina). En Actas del XVII Congreso Nacional de Arqueología Argentina (pp. 345-350), Mendoza.

Nuevo Delaunay, A., Méndez, C., Reyes O., \& Trejo, V. (2013). Evaluando evidencias humanas en la margen bosqueestepa (>900 msnm) de Patagonia central: cueva de Punta del Monte (región de Aisén, Chile). Magallania, 41(2), 127-144.

Pérez de Micou, C., \& Castro Esnal, A. (2007). Los caminos indígenas. La ruta Gan-Gan/Gastre (Chubut). En Aquí Vivieron. Arqueología y ambiente en Patagonia (pp. 9-28). Buenos Aires: AINA.

Pérez de Micou, C., Castro Esnal, A., Funes M. L., Burry L. S., \& Trivi de Mandri, M. (2009a). Prospecciones en el Río Chico, provincia de Chubut. En M. Salemme, F. Santiago, M. Alvarez, E. Piana, M. Vázquez \& E. Mansur (Eds.), Arqueología de Patagonia: una mirada desde el último confín (pp. 1149-1158). Ushuaia: Editorial Utopías.

Pérez de Micou, C., Sacchi, M., Castro Esnal A., \& Funes, M. L. (2009b). Estudios de Arqueología en la Colonia Indígena de Chalía, Dpto. Senguer, Chubut. En P. F. Azar, E. M. Cúneo \& S. N. Rodríguez (Eds.), Tras la senda de los ancestros: Arqueología de Patagonia. San Carlos de Bariloche: Universidad Nacional del Comahue.

Pérez de Micou, C., Burry, L. S., \& Trivi, M. (2009c). Imágenes desde un alero. Investigaciones multidisciplinarias en Río Mayo, Patagonia argentina. Buenos Aires: Fundación de Historia Natural Félix de Azara.

Pérez de Micou, C., Castro Esnal, A., \& Sacchi, M. (2013). Estudios preliminares en el sitio Casa de Piedra, Estancia Roselló, sudoeste de Chubut. En A. F. Zangrando, R. Barberena, A. Gil, G. Neme, M. Giardina, L. Luna, C. Otaola, S. Paulides, L. Salgán \& A. Tivoli (Eds.). Tendencias teórico-metodológicas y casos de estudio en la arqueología de la Patagonia (pp. 213-218). Mendoza: Edición del Museo de Historia Natural de San Rafael.

Pérez de Micou, C., Castro Esnal, A., Casanueva, M. L., \&
Sacchi, M. (2014a). Estudios arqueológicos en Aldea Beleiro, SO del Chubut, Argentina. IX Jornadas de Arqueología de la Patagonia. Disponible en http://www.saantropologia.com.ar/wp-content/ uploads/2014/12/PosterCoyaique.pdf

Pérez de Micou, C., Castro Esnal, A., \&. García, S. (2014b). El Corral de los Tehuelches de Loma Redonda (Chubut, Argentina). Libro de Resúmenes IX Jornadas de Arqueología de la Patagonia (pp. 64). Coyhaique: CIEP

Reyes, O., Méndez, C., Trejo, V., \& Velásquez, H. (2007). El Chueco 1: un asentamiento multicomponente en la estepa occidental de Patagonia Central (11400 a 2700 años cal AP, 44 S). Magallania, 35(1), 61-74.

Reyes, M., Peralta González, S., \& López Ferrer A. L. (2013). Análisis preliminar de los materiales líticos del sitio Delta Vulcana 1 (lago Musters, Chubut). En A. F. Zangrando, R. Barberena, A. Gil, G. Neme, M. Giardina, L. Luna, C. Otaola, S. Paulides, L. Salgán \& A. Tivoli (Eds.), Tendencias teórico-metodológicas y casos de estudio en la arqueología de la Patagonia (pp. 219-226). Mendoza: Edición del Museo de Historia Natural de San Rafael.

Reyes, M., \& Peralta, S. (2014). Análisis tecno-morfológico y de materias primas de los artefactos líticos del sitio laguna de la flecha 10 (lago Musters, Chubut). Magallania, 42(2), 199-210.

Reyes, M. R., Moreno, E., \& Stern, C. R. (2015). Uso de obsidianas entre cazadores-recolectores en la cuenca del lago Musters (Chubut, Argentina): una aproximación desde el análisis tecno-morfológico y geoquímico. Intersecciones, 16, 491-495.

Soriano, A. (1956). Los distritos florísticos de la Provincia Patagónica. Revista de Investigaciones Agrícolas, 10(4): 323-347.

Soriano, A., Movia, C. P., \& León, R. J. C. (1983). Deserts and Semi-Deserts of Patagonia. Temperate Deserts and Semi-Deserts of the World. En D. W. Goodall (Ed.), Ecosystems of the World. Temperate Deserts and Semi-deserts of the World (pp. 440-454). Nueva York: Elsevier.

Stern, C. R. (1999). Black Obsidian from central-south Patagonia, chemical characteristics, sources and regional distribution of artifacts. En Soplando en el viento. Actas de las III Jornadas de Arqueología de la Patagonia (pp. 221-234). Neuquén: Instituto Nacional de Antropología y Pensamiento Latinoamericano y Universidad Nacional del Comahue.

Stern, C. R. (2004). Obsidian in Southern Patagonia: 
review of the current information. En T. Civalero, P. Fernández \& G. Guraieb (Eds.), Contra viento y marea. Arqueología de Patagonia (pp. 167-176). Buenos Aires: Instituto Nacional de Antropología y Pensamiento Latinoamericano.

Stern, C. R., Gómez Otero, J., \&. Belardi, J. B. (2000). Características químicas, fuentes potenciales $y$ distribución de diferentes tipos de obsidianas en el Norte de la Provincia del Chubut, Patagonia Argentina. Anales del Instituto de la Patagonia, 28, 275-290.

Stern, Ch. R., Bellelli, C., \& Pérez de Micou, C. (2007). Sources and distribution of geologic and archaeologic samples of obsidian from Piedra Parada, North-Central Chubut,
Argentine Patagonia. En F. Morello, M. Martinic, A. Prieto \& G. Bahamonde (Eds.), Arqueología de FuegoPatagonia. Levantando piedras, desenterrando huesos...y develando arcanos (pp. 205-208). Punta Arenas: Ediciones CEQUA.

Stern, C. R., Castro Esnal, A., Pérez de Micou, C., Méndez, C., \& Mena, F. (2013). Circulación de obsidianas en Patagonia Central-Sur entre $44^{\circ}$ y $46^{\circ} \mathrm{S}$. En A. F. Zangrando, R. Barberena, A. Gil, G. Neme, M. Giardina, L. Luna, C. Otaola, S. Paulides, L. Salgán \& A. Tivoli (Eds.), Tendencias teórico-metodológicas y casos de estudio en la arqueología de la Patagonia (pp. 243-250). Mendoza: Edición del Museo de Historia Natural de San Rafael. 
